\title{
On the Barycentric Labeling of Certain Graphs
}

\author{
Saeid Alikhani and Zeynab Amirzadeh \\ Department of Mathematics, Yazd University, Yazd 89195-741, Iran \\ Correspondence should be addressed to Saeid Alikhani; alikhani206@gmail.com \\ Received 6 September 2014; Accepted 29 October 2014; Published 16 November 2014 \\ Academic Editor: Wai Chee Shiu
}

Copyright (c) 2014 S. Alikhani and Z. Amirzadeh. This is an open access article distributed under the Creative Commons Attribution License, which permits unrestricted use, distribution, and reproduction in any medium, provided the original work is properly cited.

Let $A$ be an abelian group. A graph $G$ is called $A$-magic if there exists edge labeling $l: E(G) \rightarrow A \backslash\{0\}$ such that the induced vertex set labeling $l^{+}: V(G) \rightarrow A$, defined by $l^{+}(v)=\sum_{u v \in E(G)} l(u v)$, where the sum is over all edges in $E(G)$, is a constant map. A graph $G$ is $A$-barycentric-magic (or has $A$-barycentric labeling) if $G$ is $A$-magic and also satisfies $l^{+}(v)=\operatorname{deg}(v) l\left(u_{v} v\right)$ for all $v \in V$ and for some vertex $u_{v}$ adjacent to $v$. In this paper we consider some graphs $G$ and characterize all $m \in \mathbb{N}$ for which $G$ is $\mathbb{Z}_{m}$-barycentric-magic.

\section{Introduction}

Let $G=(V, E)$ be a finite, simple, and undirected graph. Labeling for a graph is a map that takes graph elements to numbers (usually positive or nonnegative integers). Let $A$ be an abelian group (written additively). The graph $G$ is called $A$-magic if there exists labeling $l: E(G) \rightarrow A \backslash\{0\}$ such that, for each vertex $v$, the sum of values of all edges incident with $v$, denoted by $l^{+}(v)$, is a constant; that is, $l^{+}(v)=c$, for some $c \in A$. When this constant is $0, G$ is said to be $A$-zerosum magic. The integer-magic spectrum of a graph $G$ is the set $\operatorname{IM}(G)=\left\{k \in \mathbb{N}: G\right.$ is $\mathbb{Z}_{k}$-magic $\}$.

As an example, Figure 1 shows a graph which is $A$-zero sum magic, for every group $A$ (see [1]).

Let us state some easy lemmas (or observations). They are straightforward to verify and can be found in [2].

Lemma 1. A graph $G$ is $\mathbb{Z}_{2}$-magic if and only if every vertex of $G$ is of the same parity.

Lemma 2. An Eulerian graph $G$ with even size is A-magic.

Lemma 3. If $A_{1}$ is a subgroup of $A$ and graph $G$ is $A_{1}$-magic, then $G$ is A-magic.

Various authors have introduced labeling that generalizes the idea of magic square. Kotzig and Rosa [3] defined a magic labeling to be total labeling on the vertices and edges in which the labels are the integers from 1 to $|V(G)|+|E(G)|$. The sum of labels on an edge and its two endpoints is constant. In 1996 Ringel and Llado [4] redefined this type of labeling as edge-magic. Also, Enomoto et al. [5] have introduced the name super edge-magic for magic labeling in the sense of Kotzig and Rosa, with the added property that the $n$ vertices receive the smaller labels, $\{1,2, \ldots, n\}$. Lee et al. [6] defined the concept of $k$-edge magic graphs and studied it for certain graphs (see, e.g., [7]). Recently authors in [8] defined a new kind of group magicness graphs.

Here we recall the following definition.

Definition 4 (see [8]). If there exists labeling $l$ for a graph $G$, whose induced vertex set labeling is a constant map and for all $v \in V(G)$ the $\operatorname{sum} l^{+}(v)$ also satisfies $l^{+}(v)=\operatorname{deg}(v) l\left(u_{v} v\right)$ for some vertex $u_{v}$ adjacent to $v, G$ is said to be $A$-barycentricmagic.

Note that the motivation of Definition 4 is the following definition of $k$-barycentric sequence which was introduced in [9] and has already been used in graph labeling problems, specially in Ramsey theory [9-11].

Definition 5. Let $x_{1}, x_{2}, \ldots, x_{k}$ be $k$ elements of an abelian group $A$. This sequence is $k$-barycentric if there exists $j$ such that $x_{1}+x_{2}+\cdots+x_{j}+\cdots+x_{k}=k x_{j}$. The element $x_{j}$ is called a barycenter. 


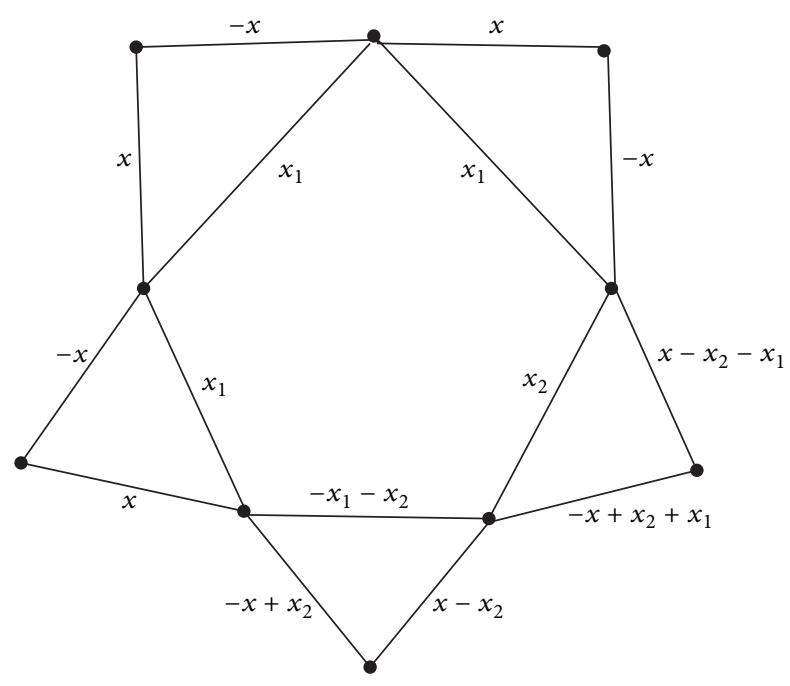

Figure 1: An example for $A$-zero sum magic graph.

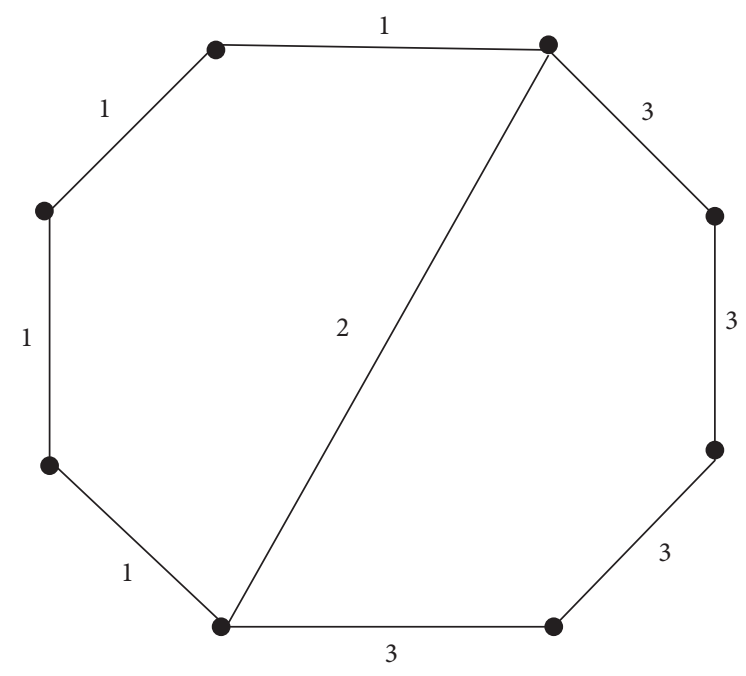

FIGURE 2: $\mathbb{Z}_{4}$-barycentric magic graph.

Example 6. Figure 2 shows a graph $G$ with $|V|=8$ and $|E|=9$ which is $\mathbb{Z}_{4}$-barycentric-magic. Note that in this example the $\operatorname{sum} l^{+}(v)$ is $2(\bmod 4)$ for all $v \in V(G)$.

The study of the barycentric-magic graphs is motivated by the relationship between different types of magic labeling and the behavior of the sums of sequences in abelian groups. That is, $A$-magic labeling of a graph $G$ is equivalent to sequences of nonzero elements of $A$ with the same sum; $A$-zero-sum magic labeling is equivalent to zero-sum sequences in $A \backslash$ $\{0\}$.

Note that $A$-barycentric-magic labeling is equivalent to sequences of nonzero elements of $A$ that contain one element which is the "average" of its terms, called barycentric sequence.
Similar to the definition of integer magic spectrum of $A$-magic graphs we state the definition of barycenter-magic spectrum of graph.

Definition 7 (see [8]). For a given graph $G$ the set of all positive integers $m$ for which $G$ is $\mathbb{Z}_{m}$-barycentric-magic is called the barycenter-magic spectrum of $G$ and is denoted by $\operatorname{BMS}(G)$.

In this paper, we consider specific graphs $G$ and characterize all $m \in \mathbb{N}$ for which $G$ is $\mathbb{Z}_{m}$-barycentric-magic.

\section{Barycentric-Magic Labeling of Certain Graphs}

In this section, we characterize all $m \in \mathbb{N}$ for which $G$ is $\mathbb{Z}_{m^{-}}$ barycentric-magic. First we consider some complete bipartite graphs.

First we state the following theorem.

Theorem 8 (see [8]). The complete bipartite graph $K_{2,3}$ is not $\mathbb{Z}_{m}$-barycentric-magic for any $m$.

We generalize the previous theorem.

Theorem 9. For $n \geq 3, K_{2, n}$ is $\mathbb{Z}_{m}$-barycentric-magic if and only if $\operatorname{gcd}(n-2, m) \neq 1$.

Proof. Let $V\left(K_{2, n}\right)=\left\{u_{1}, u_{2}\right\} \cup\left\{v_{1}, v_{2}, \ldots, v_{n}\right\}$ be the set of vertices of $K_{2, n}$. For each $j$, the edges incidents to $v_{j}$ must have the same label. Suppose that $l\left(u_{1}, v_{j}\right)=\alpha_{j}$ and $l\left(u_{2}, v_{j}\right)=\alpha_{j}$. Then $2 \alpha_{1} \equiv 2 \alpha_{2} \equiv \cdots 2 \alpha_{n}(\bmod \mathrm{m})$. We consider the two following cases.

Case 1 ( $m$ is odd). In this case, the condition $2 \alpha_{1} \equiv 2 \alpha_{2} \equiv$ $\cdots 2 \alpha_{n}(\bmod m)$ implies that all edges have the same label, say $\alpha$. By the condition $l^{+}\left(u_{i}\right)=l^{+}\left(v_{j}\right)$ we have $(n-2) \alpha \equiv$ $0(\bmod m)$ and this is impossible when $\operatorname{gcd}(n-2, m)=1$. If $\operatorname{gcd}(n-2, m)=d \neq 1$, then using $\alpha=m / d$ gives a barycentric-magic labeling.

Case 2 ( $m$ is even). Here we give two different approaches. In this case, the condition $2 \alpha_{1} \equiv 2 \alpha_{2} \equiv \cdots 2 \alpha_{n}(\bmod m)$ implies that there are at most two different labels $\alpha$ and $\beta=\alpha+m / 2$, such that $2 \alpha \equiv 2 \beta(\bmod m)$. Now label $K_{2, n}$ as follows: $l\left(u_{1}, v_{j}\right)=\alpha$ for $1 \leq j \leq k$ and $l\left(u_{1}, v_{j}\right)=\beta$ for $k+1 \leq j \leq n$, for some $1 \leq k \leq n$. Then, the edges incidents to $u_{2}$ must be labeled in the same way. This labeling is barycentric-magic if and only if

$$
k \alpha+(n-k) \beta \equiv n \alpha \equiv 2 \alpha \equiv 2 \beta(\bmod m)
$$

or

$$
k \alpha+(n-k) \beta \equiv n \beta \equiv 2 \alpha \equiv 2 \beta(\bmod m) .
$$

Without loss of generality, we consider only the first relation. The condition $n \alpha \equiv 2 \alpha(\bmod m)$ is satisfied only when $\operatorname{gcd}(n-$ $2, m) \neq 1$. So suppose that $\operatorname{gcd}(n-2, m)=d \neq 1$. Choose 


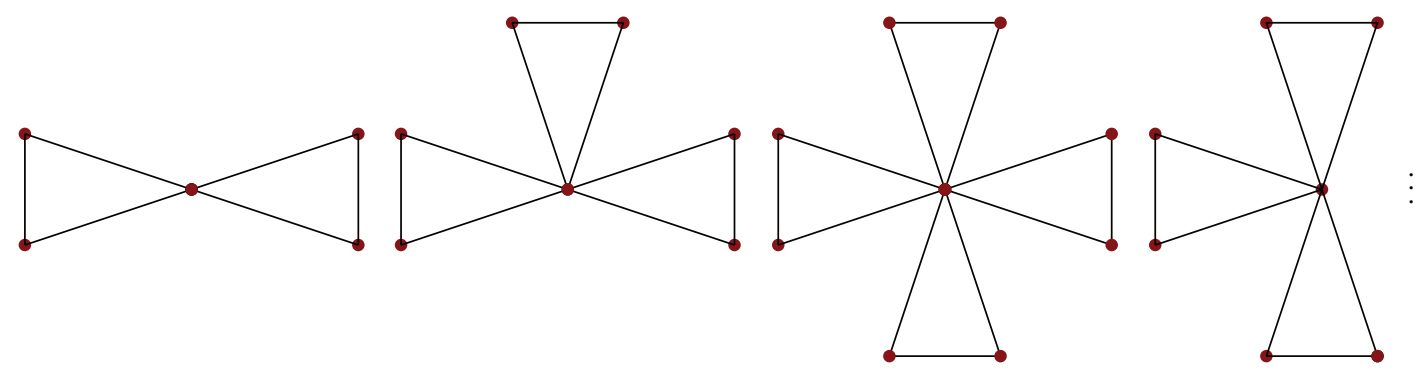

FIGURE 3: Friendship graphs $F_{2}, F_{3}, F_{4}$, and $F_{n}$, respectively.

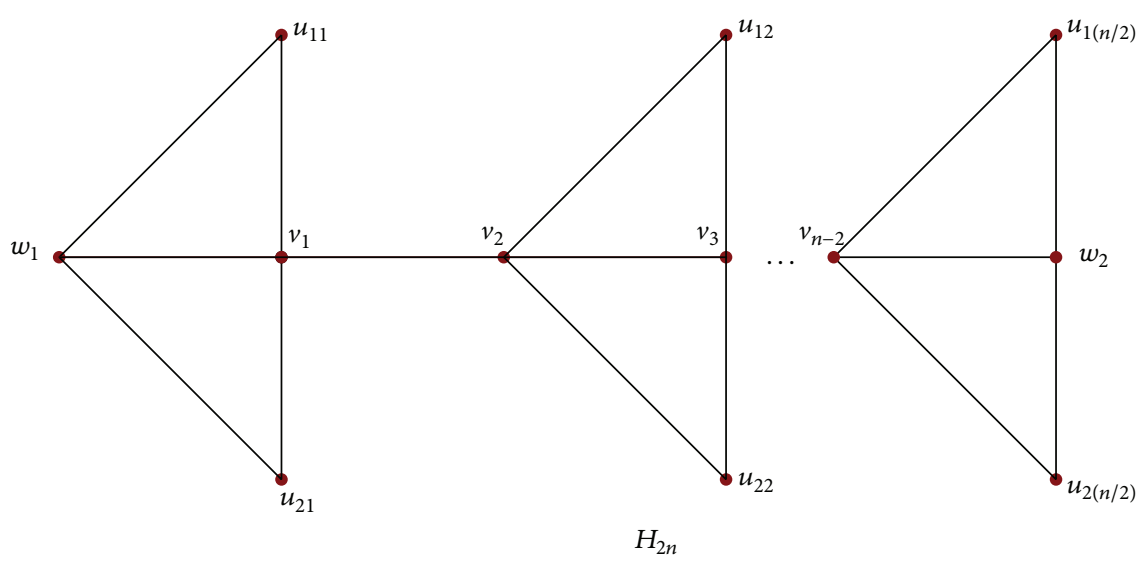

FIGURE 4: Graph $H_{2 n}$.

$k=d, \alpha=m / d$, and $\beta=\alpha+m / 2$ and since $n-d$ is even we get

$$
\begin{gathered}
k \alpha+(n-k) \beta \equiv n \alpha(\bmod m), \\
n \alpha \equiv 2 \beta \equiv 2 \alpha(\bmod m) .
\end{gathered}
$$

Therefore with this labeling $K_{2, n}$ is $\mathbb{Z}_{m}$-barycentric-magic.

Now we state the second approach.

Since $n \alpha=2 \alpha(\bmod m)$, if $\operatorname{gcd}(m, n-2)=1$, then $\alpha=0$, a contradiction. Conversely, let $\alpha_{1}=\alpha_{2}=\cdots=\alpha_{k}=m / d$. This is also a $\mathbb{Z}_{m}$-barycentric-magic labeling for $K_{2, n}$.

Example 10. Consider the graph $K_{2,10}$ and the group $\mathbb{Z}_{10}$. Let $V\left(K_{2,10}\right)=\left\{u_{1}, u_{2}\right\} \cup\left\{v_{1}, v_{2}, \ldots, v_{10}\right\}$ be the set of vertices of $K_{2,10}$. In this case, since $d=\operatorname{gcd}(n-2, m)=2$ choose $\alpha=$ $m / d=5$ and $\beta=\alpha+m / 2=10$ and $k=d=2$. The labeling of $K_{2,10}$ is as follows:

$$
\begin{aligned}
& l\left(u_{i} v_{j}\right)=5 \text { for } i=1,2 \text { and } j=1,2 ; \\
& l\left(u_{i} v_{j}\right)=10 \text { for } i=1,2 \text { and } j=3, \ldots, 10 ; \\
& \text { then } l^{+}\left(v_{j}\right)=2 \times 5=\operatorname{deg}\left(v_{j}\right) \times 5 \equiv 10(\bmod 10) \text { for } \\
& j=1,2 ; \\
& l^{+}\left(v_{j}\right)=2 \times 10=\operatorname{deg}\left(v_{j}\right) \times 10 \equiv 10(\bmod 10) \text { for } \\
& j=3, \ldots, 10 ;
\end{aligned}
$$

$$
\begin{aligned}
& l^{+}\left(u_{i}\right)=2 \times 5+8 \times 10 \equiv 10 \equiv \operatorname{deg}\left(u_{i}\right) \times 5(\bmod 10) \text { for } \\
& i=1,2 .
\end{aligned}
$$

Then with this labeling we get barycentric-magic labeling.

Here we consider friendship graphs.

Let $n$ be any positive integer and $F_{n}$ Dutch-Windmill, or friendship graph with $2 n+1$ vertices and $3 n$ edges. In other words, the friendship graph $F_{n}$ is a graph that can be constructed by coalescence $n$ copies of the cycle graph $C_{3}$ of length 3 with a common vertex. The friendship theorem of Erdös et al. [12] states that graphs with the property that every two vertices have exactly one neighbour in common are exactly the friendship graphs. Figure 3 shows some examples of friendship graphs.

Theorem 11. Friendship graphs $F_{n}$ are $\mathbb{Z}_{2 m}$-barycentric-magic graphs for any $m \in \mathbb{N}$.

Proof. Consider friendship graph $F_{n}$ and suppose that $\operatorname{deg}(v)=2 n$ and $\operatorname{deg}\left(u_{i}\right)=2$ for $i=1, \ldots, n$. We label all the edges of $F_{n}$ with $\alpha \in \mathbb{Z}_{2 m} \backslash\{0\}$. Since $2 n \alpha \equiv 2 \alpha(\bmod 2 m)$ (e.g., put $m=\alpha$ ), the graphs $F_{n}$ are $\mathbb{Z}_{2 n}$-barycentric-magic.

Here we consider another families of graphs denoted by $H_{2 n}$ (see Figure 4). 
Theorem 12. The graphs $H_{2 n}$ are not $\mathbb{Z}_{k}$-barycentric-magic for every $k$.

Proof. Suppose that the set of vertices of $H_{2 n}$ is $V=\left\{u_{1 i}, u_{2 i}\right\} \cup$ $\left\{w_{1}, w_{2}\right\} \cup\left\{v_{j}\right\}$, where $i=1, \ldots, n / 2$ and $j=1, \ldots, n-2$ and $\operatorname{deg}\left(u_{1 i}\right)=\operatorname{deg}\left(u_{2 i}\right)=2, \operatorname{deg}\left(w_{1}\right)=\operatorname{deg}\left(w_{2}\right)=3$, and $\operatorname{deg}\left(v_{j}\right)=4$. One can consider some cases to prove that there is no $\mathbb{Z}_{k}$ barycentric-magic labeling for $H_{2 n}$. Here we state two cases. We label the graph $H_{2 n}$ as follows.

Case 1. Label all edges of $H_{2 n}$ with $\alpha \in \mathbb{Z}_{k} \backslash\{0\}$. In this case $3 \alpha \equiv 2 \alpha(\bmod k)$ or $\alpha \equiv 0(\bmod k)$ which is not true.

Case 2. We label $H_{2 n}$ as follows: $l\left(w_{1} u_{11}\right)=l\left(v_{j} u_{1 i}\right)=$ $l\left(w_{2} u_{1(n / 2)}\right)=\alpha+r$ for each $i=1, \ldots, n / 2$ and $l\left(w_{1} v_{1}\right)=$ $l\left(v_{2 j} v_{2 j+1}\right)=l\left(w_{2} v_{n-2}\right)=\alpha$ for each $j=1, \ldots, n-3$ and $l\left(v_{2 j-1} v_{2 j}\right)=\alpha+3 r$ for each $j=1, \ldots, n-3$ and $l\left(w_{1} u_{21}\right)=$ $l\left(v_{j} u_{2 i}\right)=l\left(w_{2} u_{2(n / 2)}\right)=\alpha+2 r(i=1, \ldots, n / 2$ and $j=$ $1, \ldots, n-2)$. From $l^{+}\left(w_{1}\right)=l^{+}\left(u_{11}\right)$ we have $3 \alpha+3 r \equiv 2 \alpha+2 r$ $(\bmod k)$ or $\alpha+r \equiv 0(\bmod k)$, but $\alpha+r$ is the label of edges $u_{1 i} v_{j}$, so this labeling is not barycentric-magic.

\section{Conflict of Interests}

The authors declare that there is no conflict of interests regarding the publication of this paper.

\section{Acknowledgment}

The authors would like to express their gratitude to the referee for careful reading and helpful comments.

\section{References}

[1] W. C. Shiu and R. M. Low, "Integer-magic spectra of sun graphs," Journal of Combinatorial Optimization, vol. 14, no. 23, pp. 309-321, 2007.

[2] R. M. Low and S.-M. Lee, "On group-magic Eulerian graphs," Journal of Combinatorial Mathematics and Combinatorial Computing, vol. 50, pp. 141-148, 2004.

[3] A. Kotzig and A. Rosa, "Magic valuations of finite graphs," Canadian Mathematical Bulletin, vol. 13, pp. 451-461, 1970.

[4] G. Ringel and A. S. Llado, "Another tree conjecture," Bulletin of the Institute of Combinatorics and Its Applications, vol. 18, pp. 83-85, 1996.

[5] H. Enomoto, A. S. Llado, T. Nakamigawa, and G. Ringel, "Super edge-magic graphs," SUT Journal of Mathematics, vol. 34, no. 2, pp. 105-109, 1998.

[6] S.-M. Lee, H.-H. Su, and Y.-C. Wang, "On k-edge-magic Halin graphs," Congressus Numerantium, vol. 204, pp. 129-145, 2010.

[7] S. Alikhani, W. Kocay, G.-C. Lau, and S.-M. Lee, "On the $k$-edge magic graphs," Electronic Notes in Discrete Mathematics, vol. 45, pp. 35-41, 2014.

[8] S. Alikhani and B. Davvaz, "A new kind of group magicness of graphs".

[9] C. Delorme, S. Gonzalez, O. Ordaz, and M. T. Varela, "Barycentric sequences and barycentric Ramsey numbers stars," Discrete Mathematics, vol. 277, no. 1-3, pp. 45-56, 2004.

[10] C. Delorme, I. Marquez, O. Ordaz, and A. Ortuño, "Existence conditions for barycentric sequences," Discrete Mathematics, vol. 281, no. 1-3, pp. 163-172, 2004.
[11] S. González, L. González, and O. Ordaz, "Barycentric Ramsey numbers for small graphs," Bulletin of the Malaysian Mathematical Sciences Society: Second Series, vol. 32, no. 1, pp. 1-17, 2009.

[12] P. Erdös, A. Renyi, and V. T. Sos, "On a problem of graph theory," Studia Scientiarum Mathematicarum Hungarica, vol. 1, pp. 215235, 1966. 


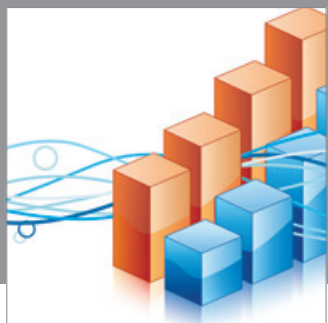

Advances in

Operations Research

mansans

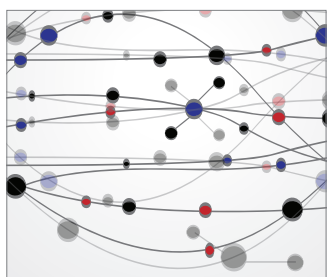

The Scientific World Journal
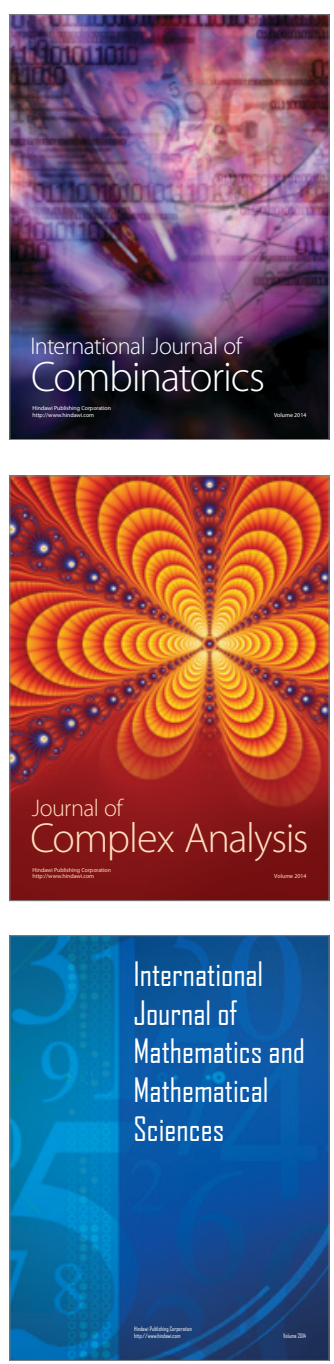
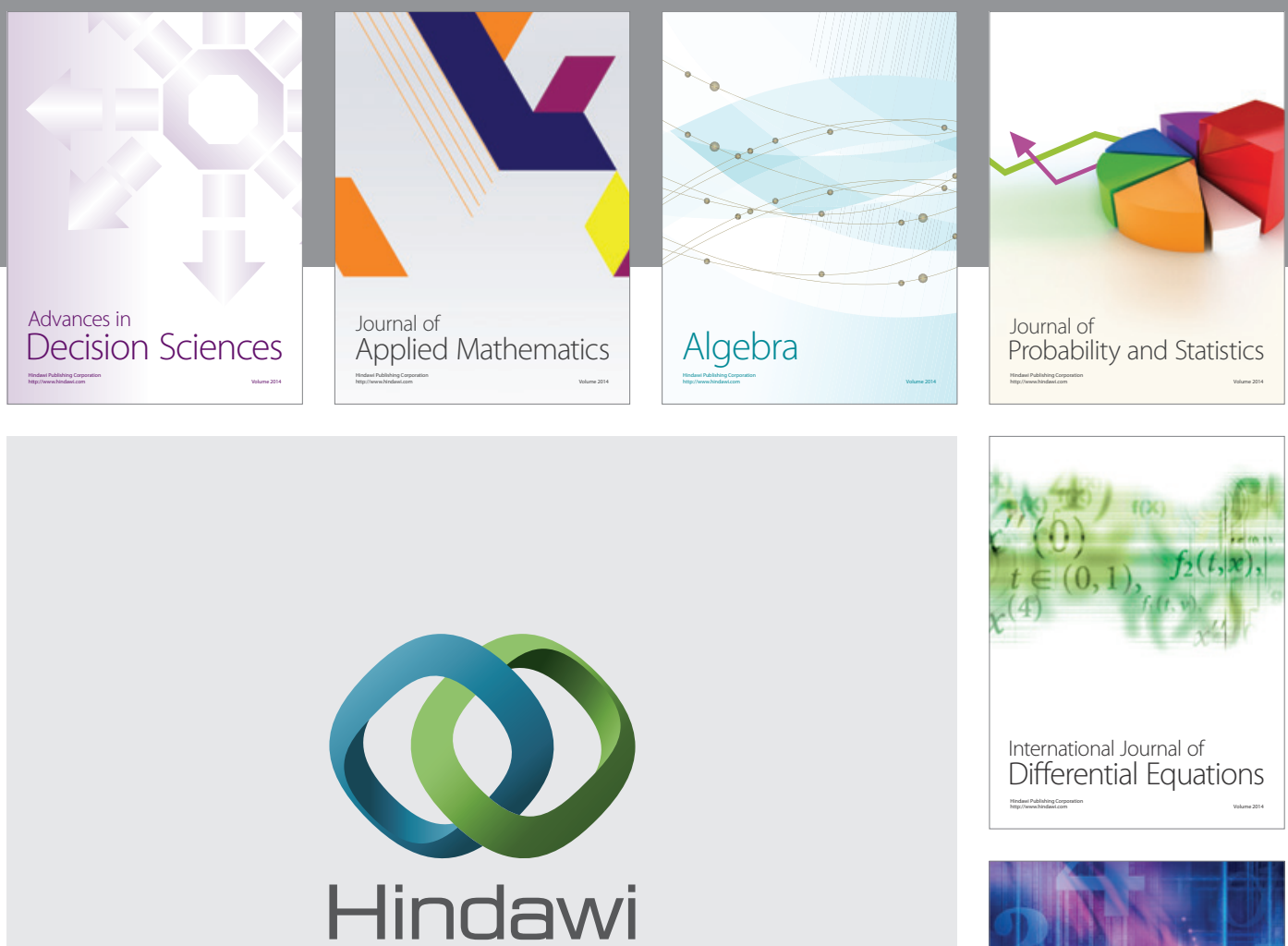

Submit your manuscripts at http://www.hindawi.com
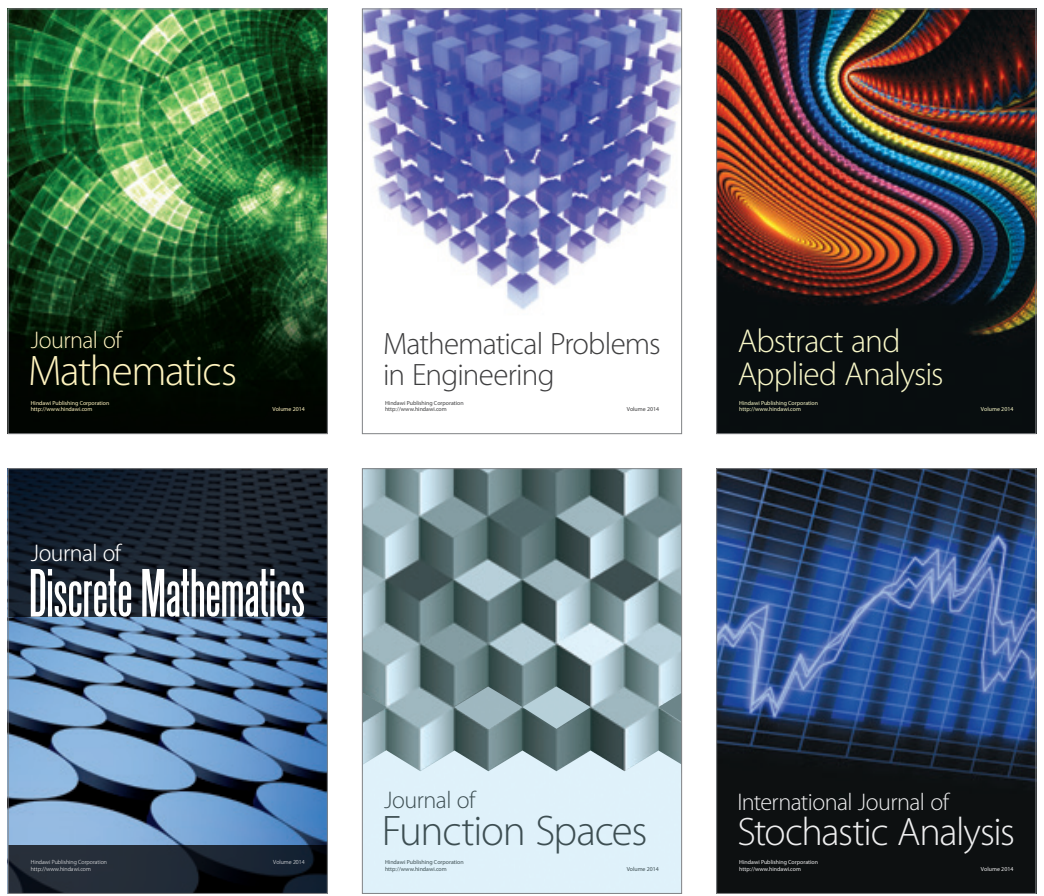

Journal of

Function Spaces

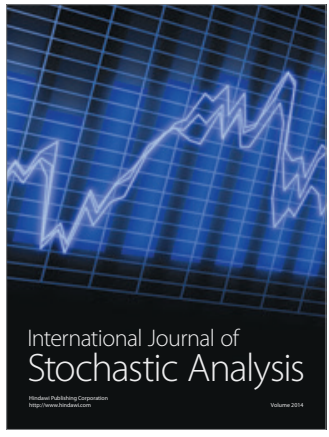

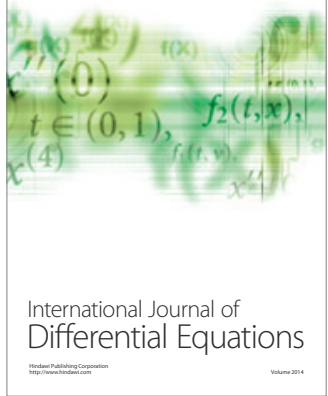
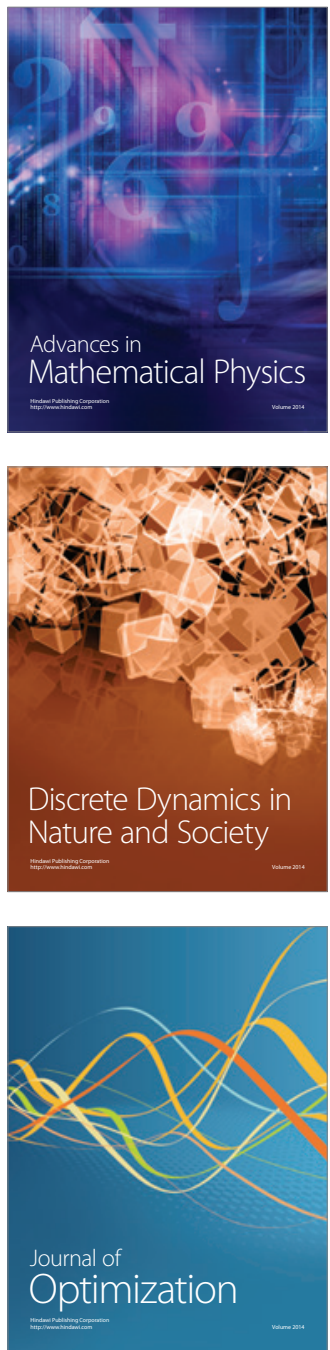